\title{
Expression of Draxin in Lung Carcinomas
}

\author{
Younosuke Sato ${ }^{1}$, Akira Matsuo ${ }^{1}$, Shinji Kudoh ${ }^{1}$, Liu Fang ${ }^{1,2}$, Koki Hasegawa ${ }^{3}$, \\ Yohei Shinmyo ${ }^{4}$ and Takaaki Ito ${ }^{1}$
}

${ }^{1}$ Department of Pathology and Experimental Medicine, Graduate School of Medical Sciences, Kumamoto University, Honjo 1-1-1, Chuo-ku, Kumamoto 860-8556, Japan, ${ }^{2}$ Department of Clinical Laboratory, Fourth Affiliated Hospital of Harbin Medical University, Harbin, China, ${ }^{3}$ Center for Instrumental Analysis, Kyoto Pharmaceutical University, Misasaginakauchiomachi 5, Yamashina-ku, Kyoto, Japan and ${ }^{4}$ Department of Biophysical Genetics, Graduate School of Medical Sciences, Kanazawa University, Takara-machi 13-1, Ishikawa 920-8640, Japan

Received December 13, 2017; accepted January 16, 2018; published online February 21, 2018

Guidance molecules, such as Netrin-1, and their receptors have important roles in controlling axon pathfinding, modulate biological activities of various cancer cells, and may be a useful target for cancer therapy. Dorsal repulsive axon guidance protein (Draxin) is a novel guidance molecule that binds not only common guidance molecule receptors with Netrin-1, but also directly binds the EGF domain of Netrin-1 through a 22-amino-acid peptide (22aa). By immunostaining, Draxin was positively expressed in small cell carcinoma, adenocarcinoma (ADC), and squamous cell carcinoma of the lung. In addition, western blot analysis revealed that Draxin was expressed in all histological types of lung cancer cell lines examined. Knockdown of Draxin in an ADC cell line H358 resulted in altered expression of molecules associated with proliferation and apoptosis. The Ki-67 labeling index of Draxinknockdown ADC cells was increased compared to that of control ADC cells. In H358 cells, treatment of 22aa induced phosphorylation of histone H3, but did not change apoptosisassociated enzymes. These data suggest that Draxin might be involved in cell proliferation and apoptosis in lung adenocarcinoma cells.

Key words: lung cancer, Draxin, Netrin-1, dependence receptor, cell proliferation

\section{Introduction}

Guidance molecules have crucial roles in controlling axon pathfinding by which neurons can extend axons to reach their correct targets. Guidance molecules work as a cue to the growth cone, or growing axon tip, from the extracellular environment and can attract or repel axons through surface receptors, intracytoplasmic signaling pathways, and cytoskeletal changes. Guidance molecules, such as Netrin, Ephrin, Slit and Semaphorin (Sema), and their receptors have been identified and the molecular basis of axon pathfinding has been established $[9,33,36]$. Dorsal

Correspondence to: Takaaki Ito, MD, DMSc, Department of Pathology and Experimental Medicine, Kumamoto University, Graduate School of Medical Sciences, Honjo 1-1-1, Chuo-ku, Kumamoto 860-8556, Japan. E-mail: takaito@kumamoto-u.ac.jp repulsive axon guidance protein (Draxin) was discovered by signal sequence trap screening of the chick embryonic brain [18]. Draxin is a novel guidance molecule, and inhibits or repels neurite outgrowth from dorsal spinal cord and cortical explants in vitro [18]. Guidance molecules were originally described as controlling axon navigation in the developing brains, but they can also work in branching morphogenesis of the epithelial system and in the development of the vascular network $[16,17,32]$. During fetal lung development, Sema3A inhibits branching morphogenesis via its receptor neuropilin 1, but other class 3 Semas, such as Sema3C and Sema3F, stimulate branching morphogenesis and cell proliferation $[19,20]$. Another guidance molecule Netrin-1 is detected immunohistochemically in the epithelial and mesenchymal tissues of fetal mouse lungs [7], and Netrin-1 and Netrin-4 are have roles in the lung 
epithelial bud by modulating the morphogenetic response of the lung endoderm to exogenous fibroblast growth factors [25]. Netrin receptors include two main protein families: Deleted in Colorectal Cancer (DCC) and Neogenin, and Uncoordinated family member 5 orthologues (UNC5) $[3,23]$. These receptors are spatially and temporarily expressed in fetal developing lungs [7, 13, 25], and orchestrate the development of lungs along with guidance molecules.

Besides roles in development, guidance molecules and their receptors have been reported to modulate biological activities of various cancers cells including lung cancers [3, $28,30]$. Netrin-1 can stimulate the migration of human melanoma, glioblastoma, and pancreatic adenocarcinoma cells $[10,22]$. Netrin-1 and its receptors are important for angiogenesis $[6,27]$, and the proangiogenic effects of Netrin-1 were reported in pancreatic cancer cell lines [10]. DCC, a Netrin receptor, was first identified as a candidate tumor-suppressor gene in colorectal cancer [11], and DCC may be a ligand-dependent suppressor [3]. When Netrin-1 binds DCC on the cell membrane, signals can lead to cell proliferation and migration. However, in the absence of Netrin-1, DCC signaling induces apoptosis $[3,4,28]$. The mechanisms of induced apoptosis in the absence of ligands is obscure, but could result from proapoptotic effectors such as death-associated protein kinase and caspase 9 [26, 28, 29]. Because interaction between Netrin-1 and its receptors can regulate cancer cell survival, inhibition of this ligand-receptor system may be a useful target for cancer therapy [28]. In lung adenocarcinomas, transfection of siRNA for Netrin-1 enhanced lung adenocarcinoma cell death. The fibronectin domain of DCC can work as a decoy protein to inhibit Netrin-1 and DCC signaling and to induce apoptosis [8].

Phenotypic similarities have been reported in Netrin-1 and Draxin-deficient mice [18, 31], which suggests some common receptor-signaling system involving both ligands. Draxin can bind to Netrin receptors such as DCC, Neogenin, and UNC5 [1], but the binding sites on these receptors may be different. Netrin-1 binds the fibronectin domain of DCC and Neogenin [34], but Draxin binds the immunoglobulin domain of DCC and Neogenin [1]. Recently, Draxin was shown to bind a 22-amino-acid peptide (22aa) of the EGF domain of Netrin-1, and through Draxin/Netrin-1 interaction, Draxin may act as a secreted Netrin-1 antagonist [15]. Because of the significant role of Netrin-1 and its binding receptors in various cancer cells including lung cancer, the expression and biological significance of Draxin should be investigated. In the present study, expression of Draxin was examined in surgically resected lung cancer tissues and human lung cancer cell lines. Draxin-knockdown experiments and Draxin 22aa treatment were performed to reveal the biological significance of Draxin in a lung adenocarcinoma cell line. Furthermore, a mouse lung carcinogenesis study was performed in Draxin-deficient mice.

\section{Materials and Methods}

\section{Tissue samples}

Lung cancer tissue samples of small cell carcinoma ( $S C L C ; n=11)$, adenocarcinoma (ADC; $n=36$ ), and squamous cell carcinoma (SCC; $\mathrm{n}=23$ ), and normal lung tissues from the lung cancer files of the Department of Pathology and Experimental Medicine of Kumamoto University and from the Department of Thoracic Surgery of Kumamoto University were used. Histological diagnosis of the samples was made by the World Health Organization's criteria. The study followed the guidelines of the Ethics Committee of Kumamoto University.

\section{Immunohistochemical analysis}

Formalin-fixed, paraffin-embedded specimens were cut into sections ( $4 \mu \mathrm{m}$ thick) and mounted onto MAS-GPcoated slides (Matsunami Glass Ind, Osaka, Japan). After deparaffinization and rehydration, sections were heated using an autoclave in $0.01 \mathrm{~mol} / \mathrm{L}$ citrate buffer $(\mathrm{pH} 7.0)$ for antigen retrieval. Sections were incubated with $0.3 \% \mathrm{H}_{2} \mathrm{O}_{2}$ in absolute methanol for 30 min to block endogenous peroxidase activity. Next, sections were incubated with 5\% skim milk for 20 min to block non-specific binding. After this blocking step, sections were incubated with primary antibody (Table 1) at $4^{\circ} \mathrm{C}$ overnight or for $70 \mathrm{~min}$ at room temperature. This was followed by a 1-hour incubation with secondary antibody (En Vision+ System-HRP-Labeled Polymer; Dako (Agilent), CA, USA) and visualization with Liquid DAB + Substrate Chromogen System (Dako). All slides were counterstained with hematoxylin for $30 \mathrm{sec}$ before dehydration and mounting. The specificity of immunolabeling of each antibody was tested using normal mouse IgG (Santa Cruz Biotechnology, CA, USA) and normal rabbit IgG (Santa Cruz Biotechnology), and no staining was observed.

\section{Cell lines}

Seven SCLC cell lines (H69, H889, H69AR, SBC1, H1688, SBC3, SBC5), three ADC cell lines (H358, A549, H1975), and three SCC cell lines (H226, H2170, H15) were used. H69, H889, H69AR, SBC1, H1688, H358, A549, H1975, H2170, and H226 were purchased from ATCC (Manassas, VA, USA), and $\mathrm{SBC} 1, \mathrm{SBC} 3$, and $\mathrm{SBC} 5$ were purchased from the Japan Collection of Research Bioresources Cell Bank (Osaka, Japan). H15 was a generous gift from Dr. Adi F. Gazdar (University of Texas Southwestern Medical Center, Dallas, TX, USA).

\section{Transfection with siRNA}

Draxin siRNA (sc-88131, Santa Cruz) and control siRNA (sc-37007, Santa Cruz) were transfected into an adenocarcinoma cell line $\mathrm{H} 358$ at a concentration of 20 $\mathrm{nmol} / \mathrm{L}$ using an electroporator (NEPA21 pulse generator; Nepa Gene, Chiba, Japan) as described in the manufacturer's instructions. Cells were harvested $72 \mathrm{hr}$ after 
Table 1. Antibodies used for $W B$ and IHC analyses

\begin{tabular}{lllc}
\hline \multicolumn{1}{c}{ Primary antibody } & \multicolumn{1}{c}{ Manufacturer (location) } & WB & IHC (ICC) \\
\hline Draxin (C-term) & Acris (Germany) & $1: 2000$ & $1: 300$ \\
Draxin (AF6148) & R\&D (USA) & $1: 1000$ & $1: 1000$ \\
Netrin-1 (NTN1) & LSBio (USA) & $1: 500$ \\
Netrin-1 & Bioss (USA) & $1: 1000$ & $1: 250$ \\
Neogenin (NEO1) & Abnova (Taiwan) & $1: 500$ & $1: 500$ \\
DCC & Acris & & $1: 5000$ \\
DCC & Millipore (USA) & $1: 1000$ & \\
Akt & Cell Signaling Technology (USA) & $1: 5000$ & \\
Phosphorylated-Akt (Ser473) & Cell Signaling Technology & $1: 500$ & \\
Caspase-3 (9662) & Cell Signaling Technology & $1: 1000$ & $1: 1000$ \\
Cleaved Caspase-3 (9661) & Cell Signaling Technology & $1: 1000$ & \\
Caspase-8 (9746) & Cell Signaling Technology & $1: 5000$ \\
Caspase-9 (9508) & Cell Signaling Technology & $1: 1000$ \\
Bcl-2 (50E3) & Cell Signaling Technology & $1: 500$ \\
Phosphorylated-Bcl-2 (5H2) & Cell Signaling Technology & $1: 20,000$ \\
CyclinD1 (SP4) & NeoMarkers (USA) & \\
Phosphorylated-Histone H3 (Ser10) & Millipore & & $(1: 50)$ \\
$\beta$-actin (A-5441) & Sigma Aldrich (USA) & & \\
Ki-67 & Dako (USA) & & \\
\hline
\end{tabular}

ICC, immunocytochemistry; IHC, immunohistochemistry; WB, western blot.

transfection and separated for Ki-67 immunostaining by centrifugation. Collected cells were heated using an autoclave in $0.01 \mathrm{~mol} / \mathrm{L}$ citrate buffer $(\mathrm{pH} 9.0)$ for antigen retrieval. The methods after antigen retrieval were the same as the immunostaining methods described above. We observed about 1000 cells using a microscope and calculated the cell death rate.

\section{Statistical analysis}

All statistical analyses were performed with EZR (Saitama Medical Center, Jichi Medical University, Saitama, Japan), which is a graphical user interface for $\mathrm{R}$ (The $\mathrm{R}$ Foundation for Statistical Computing, Vienna, Austria) [21]. More precisely, it is a modified version of $\mathrm{R}$ commander designed to add statistical functions frequently used in biostatistics.

\section{WB analysis}

Lung cancer cell lines were used for WB analysis. Primary antibodies used for WB analysis are listed in Table 1. The membrane was washed and incubated with secondary antibody conjugated with horseradish peroxidase for $1 \mathrm{hr}$, and the immune complex was visualized with a chemiluminescence substrate (Amersham Pharmacia Biotech, Buckinghamshire, UK).

\section{Synthesis of Biotin-Draxin 22-amino-acid peptide (22aa)}

NH2-PEG2-Suc-Gly-Glu(OtBu)-Val-Met-Pro$\operatorname{Thr}(\mathrm{tBu})$-Leu-Asp(OtBu)-Met-Ala-Leu-Phe-Asp(OtBu)$\operatorname{Trp}(\mathrm{Boc})-\mathrm{Thr}(\mathrm{tBu})-\mathrm{Asp}(\mathrm{OtBu})-\mathrm{Tyr}(\mathrm{tBu})-\mathrm{Glu}(\mathrm{OtBu})-$ Asp(OtBu)-Leu-Lys(Boc)-Pro was assembled from RINKChemMatrix resin using the standard Fmoc protocol. A mixture of Biotin-OSu and DIPEA in DMF was added to the protected peptide resin to produce a biotin-modified derivative. The resin was washed with methanol and dried in vacuo. An aliquot of the resin was treated with a mixture of TFA/water/ethanedithiol/triisopropylsilane (94:2.5:2.5:1) for $2 \mathrm{hr}$, followed by precipitation in cold dimethyl ether. The crude peptide was purified by HPLC on a Cosmosil 5 C18 ARII column $(4.6 \times 250 \mathrm{~mm})$ with two solvent systems and the gradient elution method at a flow rate of 1 $\mathrm{mL} / \mathrm{min}$. Solvent A was $0.1 \%$ TFA in water, and solvent B was $0.1 \%$ TFA in acetonitrile. After lyophilization, BiotinDraxin22 was obtained as a white powder. Draxin 22aa was measured by matrix-assisted laser desorption/ionization time-of-flight mass spectroscopy. The calculated mass for $(\mathrm{M}+\mathrm{H})+$ was 3041.40. The concentration analysis of Draxin 22aa was quantified using Quant*Tag Biotin kit (VECTOR Laboratories, CA, USA).

\section{Draxin 22a treatment in the $\mathrm{H} 358$ adenocarcinoma cell line}

H358 adenocarcinoma cells were seeded onto a 6-well plate. At 50\% confluency, medium was replaced with medium plus Draxin 22aa. The concentration of the peptide was prepared at $1 \mu \mathrm{M}, 10 \mu \mathrm{M}$, and $100 \mu \mathrm{M}$. After $\mathrm{H} 358$ cells were incubated for 2 days at $37^{\circ} \mathrm{C}$, cells were collected for WB analysis.

\section{Mouse lung tumorigenesis study}

To understand the significance of Draxin in a mouse lung tumorigenesis model, we treated wild-type and Draxin gene-deficient mice with 4-(methylnitrosamine)-1(3-pyridyl)-1-butanone (NNK) (Toronto Research Chemicals, Toronot, Canada). Male and female heterozygous Draxin gene-deficient mice were mated, and wild-type and homozygous Draxin gene-deficient mice were obtained. Animals were maintained with free access to water and food on a $12 \mathrm{hr}$ light/dark cycle under pathogen-free condi- 


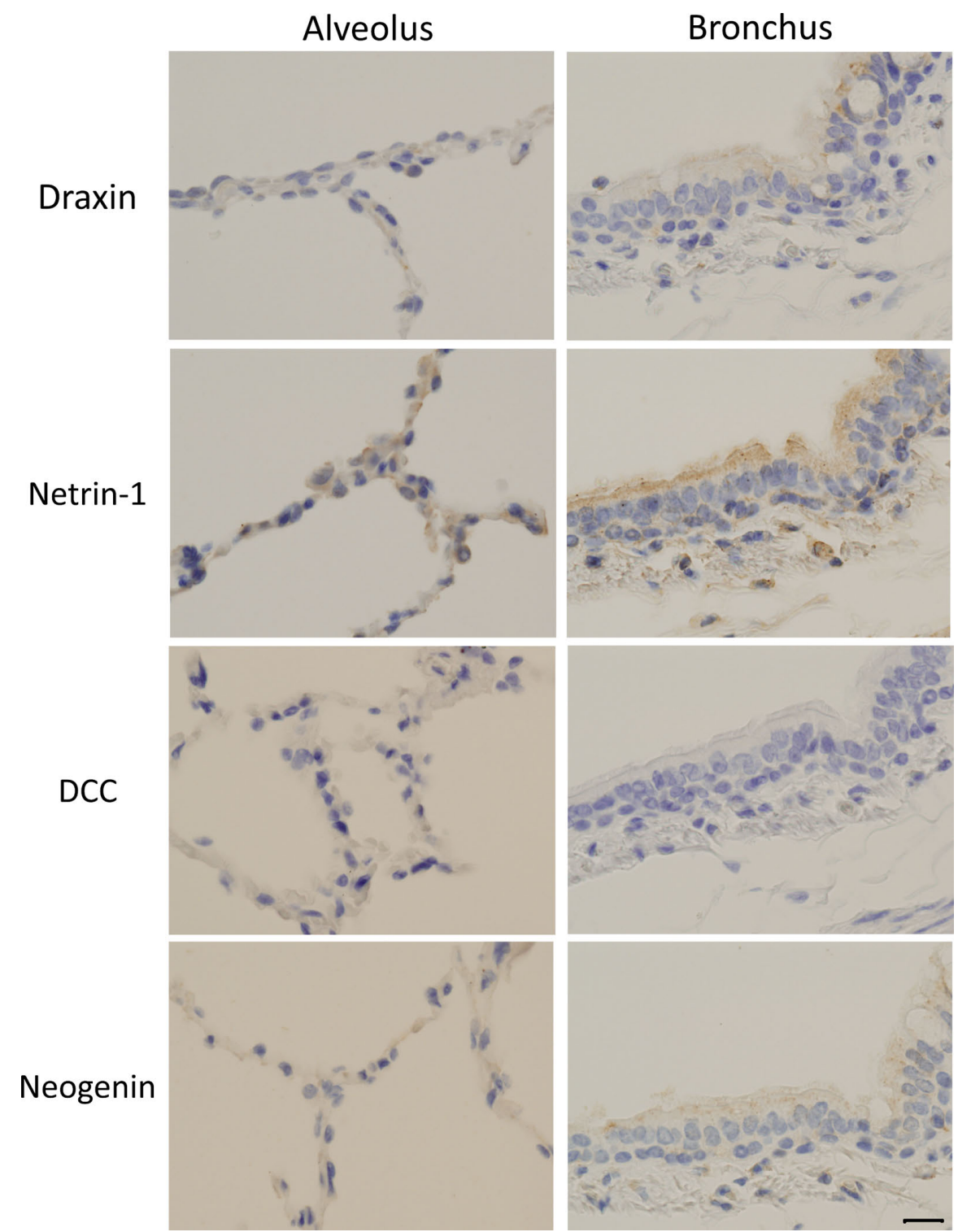

Fig. 1. Immunohistochemical analysis of the expression of Draxin, Netrin-1, and their receptors, DCC and Neogenin, in normal lung tissue. The surface of alveolar and bronchiolar epithelial cells was positively stained fot Netrin-1. Draxin and dependence receptors were weakly or poorly stained in alveolar and bronchiolar epithelial cells. Bar $=20 \mu \mathrm{m}$.

tions. Genotyping of the mice was performed according to our previous study [18]. Ten male wild-type and homozygous Draxin gene-deficient mice at 10 weeks old were treated with an intra-peritoneal injection of NNK (100 $\mathrm{mg} / \mathrm{kg}$ body weight) once-a-week for 2 weeks, and sacrificed 40 weeks after the first injection. The lung tissues were injected via the trachea with $4 \%$ paraformaldehyde in phosphate buffer solution ( $\mathrm{pH}$ 7.3). Lung tissue slices including tumors were fixed in fixative for one week, washed, and embedded in paraffin. Paraffin sections were stained with hematoxylin and eosin, and tumors were histologically evaluated according to the criteria of Foley to discriminate adenomas and adenocarcinomas [14]. This study was approved by the Animal Care Committee of Kumamoto University (\#27-147).

\section{Results}

\section{Draxin and receptors in lung cancer (SCLC, ADC, SCC) tissue sections}

First, expression of Draxin, Netrin-1, and their receptors (Neogenin, DCC) was examined in normal tissue (Fig. 1) and lung cancer sections (Fig. 2) by immunohistochemistry. These samples include 11 SCLC cases, 36 ADC cases, 23 SCC cases, and normal lung tissue (Table 2). In normal lung tissue, weak expression of Draxin was observed. In contrast, Netrin-1 was expressed moderately in normal lung tissue. DCC and Neogenin were weakly or poorly expressed. In lung cancer tissues, Draxin was strongly expressed in SCLC, ADC, and SCC. The expression of Draxin in SCLC was weaker than that in ADC and 


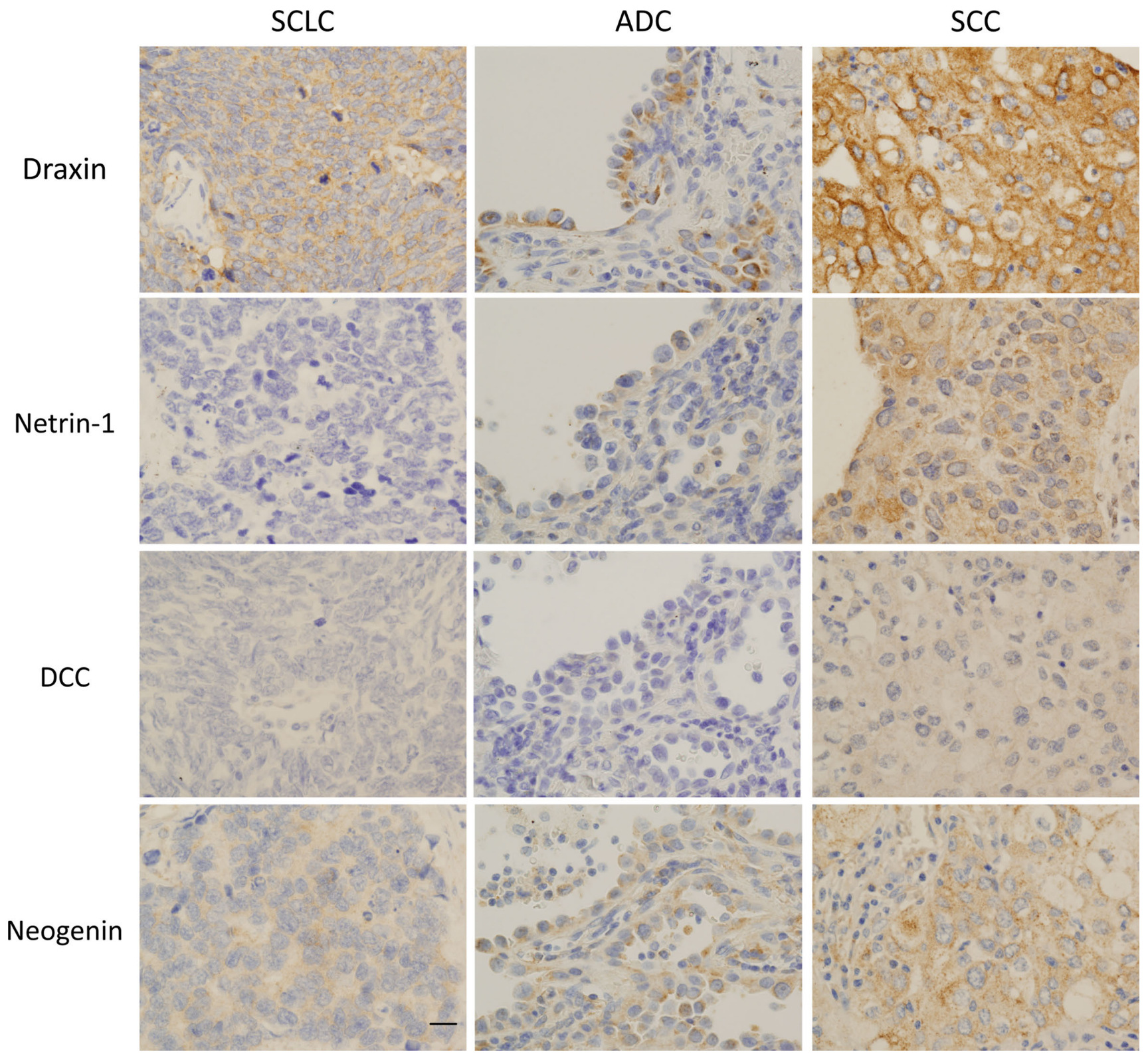

Fig. 2. Immunohistochemical analysis of the expression of Draxin, Netrin-1, and their receptors, DCC and Neogenin, in lung cancer tissue. Draxin was positively stained in all types of lung cancer, especially in ADC and SCC. Draxin was clearly expressed in lung cancer compared with that of Netrin-1 and receptors. $\mathrm{Bar}=20 \mu \mathrm{m}$.

Table 2. Immunohistochemical analysis in lung cancer tissue

\begin{tabular}{|c|c|c|c|c|c|}
\hline & $\mathrm{n}$ & Draxin & Netrin-1 & DCC & Neogenin \\
\hline SCLC & 11 & $5(45.4 \%)$ & $5(45.4 \%)$ & $0(0 \%)$ & $7(63.6 \%)$ \\
\hline $\mathrm{ADC}$ & 36 & $32(88.9 \%)$ & $11(30.6 \%)$ & $9(25.0 \%)$ & $24(66.7 \%)$ \\
\hline $\mathrm{SCC}$ & 23 & $21(91.3 \%)$ & $20(87.0 \%)$ & $15(65.2 \%)$ & $20(87.0 \%)$ \\
\hline
\end{tabular}

SCC. Netrin-1 was poorly expressed in SCLC and ADC. The expression of Netrin-1 was observed in SCC. In addition, Neogenin was strongly expressed in SCC. The expression of DCC was weakly observed in SCLC and SCC.

\section{Draxin and receptors were expressed in some lung cancer cell lines}

The expression of Draxin, Netrin-1, and their receptors in lung cancer cell lines was examined by WB analysis (Fig. 3). WB analysis was performed in SCLC (H69, H889, 


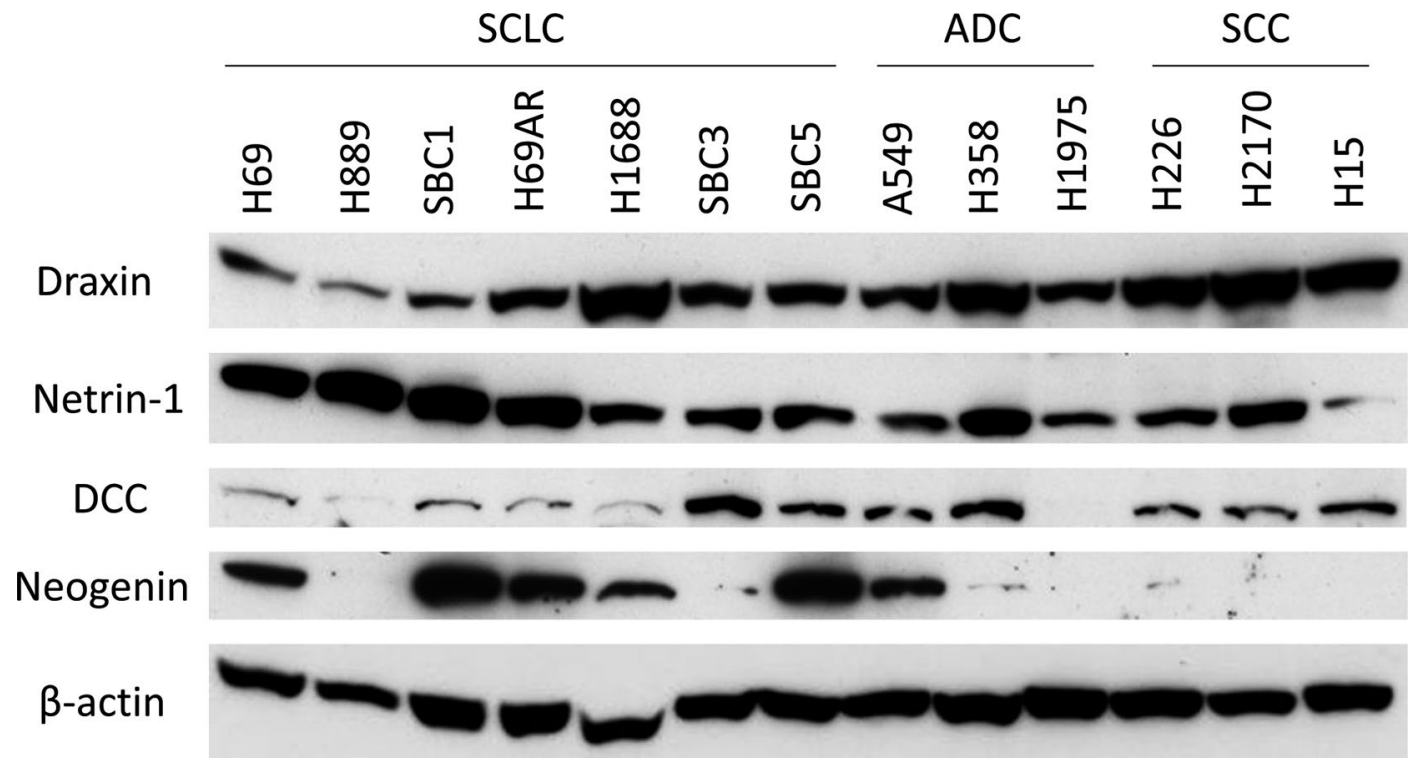

Fig. 3. Western blot analysis of Draxin, Netrin-1, and their receptors, DCC and Neogenin, in lung cancer cell lines. Western blot analysis was performed using 13 human lung cancer cell lines including seven SCLC, three ADC, and three SCC cell lines. $\beta$-actin served as an internal control.

SBC1, H69AR, H1688, SBC3, and SBC5), ADC (A549, $\mathrm{H} 358$, and H1975), and SCC (H226, H2170, and H15). Draxin was detected and evenly expressed in all types of lung cancer cell lines, especially in H69AR, H358, H226, H2170, and H15. Netrin-1 was also detected in lung cancer cells. For Neogenin, expression was detected in SCLC more than in ADC, and poorly detected in SCC. DCC was only modestly or slightly expressed in all types of lung cancer cells. Collectively, these results indicate that axon guidance molecules exist in lung cancer regardless of tissue type, but the presence of DCC and Neogenin in lung cancer cells depends on histological type.

\section{The suppression of Draxin in H358 induced a decrease in apoptosis-related proteins and increased phosphorylated- histone $\mathrm{H3}$ (ser10).}

WB analysis of H358, an adenocarcinoma cell line, was performed after Draxin knockdown to investigate the role of Draxin in apoptosis and proliferation. We used an antibody against phosphorylated-histone H3 (ser10) as a mitotic marker, antibodies against Caspase3, cleaved caspase3 (cCASP3), Caspase8, Caspase9, B-cell lymphoma 2 (Bcl-2) as apoptosis-related markers, and antibodies against AKT and phosphorylated-AKT as markers of cell signaling activity. Results are shown in Fig. 4. All markers had a similar expression pattern between control siRNA and nontreated cells in H358 cells. Suppression of Draxin reduced Caspase3, Caspase8, and Caspase9 expression and elevated phosphorylated-histone $\mathrm{H} 3$. The average $\mathrm{Ki}-67$ index in H358 cells with reduced Draxin expression was $63.6 \%$, and was elevated around $20 \%$ compared with that of $\mathrm{H} 358$ siRNA-control cells.
The role of Draxin 22a in $\mathrm{H} 358$ cells.

WB analysis of H358 cells cultured with Draxin 22aa peptide was performed to reveal the relationship between the peptide, Netrin-1, proliferation, and apoptosis. Expression of phosphorylated-histone H3 increased in H358 cells treated with increasing amounts of Draxin 22aa peptide. Other protein expression was not significantly altered in H358-treated cells (Fig. 5).

\section{Mouse lung tumorigenesis study}

Lung tumors including adenoma and adenocarcinoma were observed in Draxin gene-deficient and wild-type mice (Fig. 6). Incidence of the lung tumors and the ratio of adenocarcinoma to adenoma were similar in both types of mice (Table 3 ).

\section{Discussion}

Draxin inhibits axonal outgrowth and is involved in the formation of forebrain commissures [18], which is mediated by Draxin receptors [1]. Binding of guidance proteins to receptors, such as DCC, affects cell survival, and the relationship between guidance proteins and cancer cell survival is a potential target for cancer therapies $[26,28$, 29]. The role of Netrin-1 in cancer has been investigated, but the role of Draxin, which shares receptors with Netrin, in cancer has never been studied. In the present study, we demonstrated that Draxin is expressed in lung cancer cell lines and tissues by IHC and WB analyses. IHC revealed that Draxin is expressed in lung cancer cells more than in normal lung epithelial cells. This observation suggests that Draxin may be an important factor to regulate receptor signaling in lung cancer cells. In addition, WB analysis 


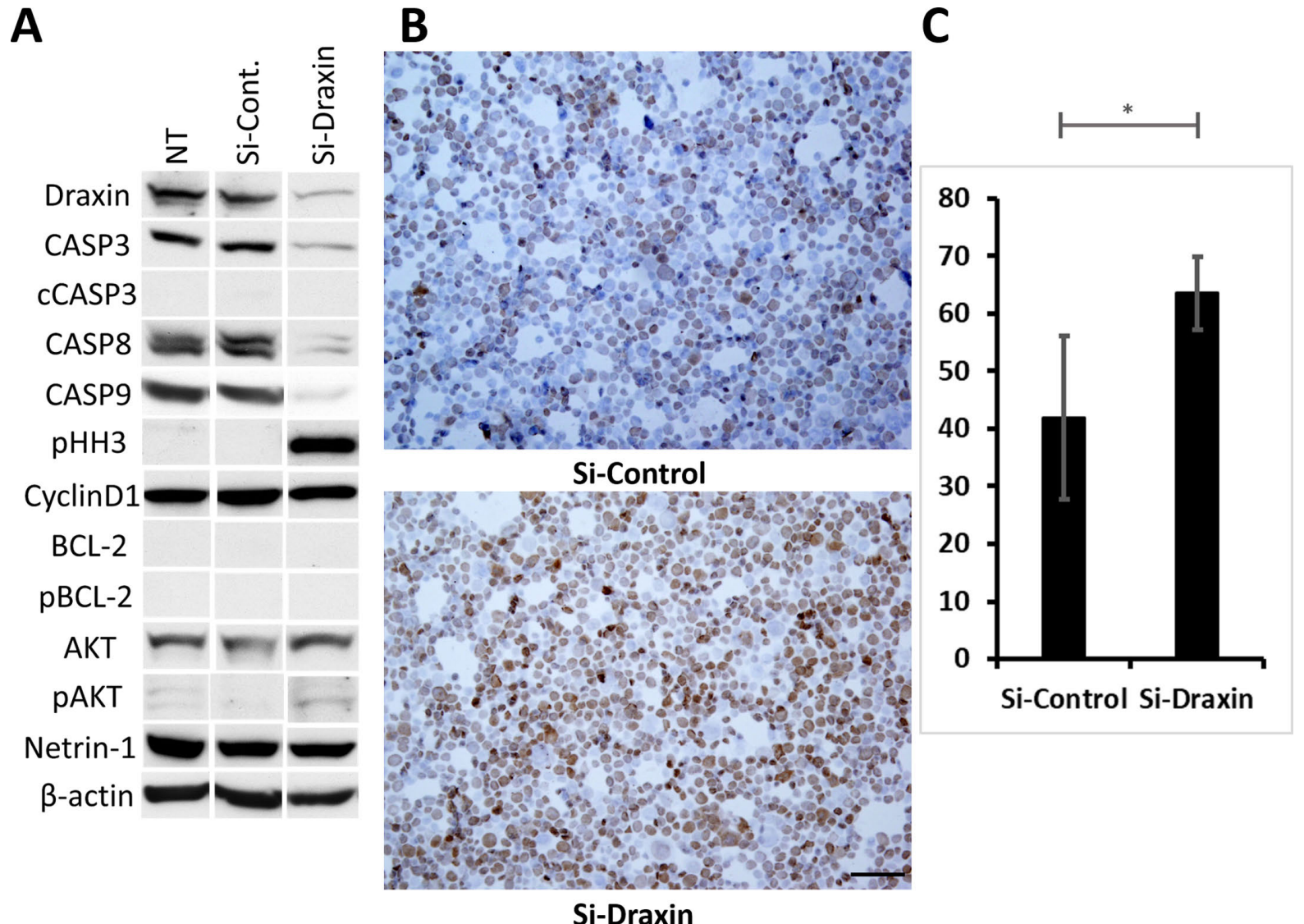

Fig. 4. Evaluation of the relationship between Draxin and tumorigenesis. (A) Western blot analysis of Draxin knockdown in human lung adenocarcinoma cells (H358). Three biological replicates were analyzed. Representative examples of no treatment (NT), transfected control siRNA (Si-cont.), and Draxin siRNA (Si-Draxin) are shown. Expression of phosphorylated-histone H3 (ser10) and CyclinD1 as mitotic markers, antibodies against Caspase3 (CASP3), Cleaved Caspase3 (cCASP3), Caspase8 (CASP8), Caspase9 (CASP9), B-cell lymphoma 2 (Bcl-2), and phosphorylated-Bcl-2 (pBcl-2) as apoptosis-related markers, and antibodies against AKT and phosphorylated-AKT (pAKT) as markers of cell signaling activity. Draxin knockdown was successfully performed in Si- Draxin cells. All markers showed similar expression patterns between NT and Si-cont. Draxin siRNA induced the suppression of CASP3, CASP8 and CASP9. In addition, Draxin siRNA elevated of pHH3 levels. $\beta$-actin served as an internal control. (B) Ki-67 (MIB-1) index analysis of Draxin knockdown in human lung adenocarcinoma cell (H358). Upper panel, transfected control siRNA (control) where a moderate number of adenocarcinoma cells were positive for Ki-67. Lower panel, Draxin siRNA (Si-Draxin) where most adenocarcinoma cells were positive for $\mathrm{Ki}-67$. Bar $=100 \mu \mathrm{m}$. (C) The average Ki-67 index in transfected control siRNA cells was 41.9\%. The average Ki-67 index in Draxin siRNA cells was $63.6 \%$. $* x<0.01$.

showed that Draxin is expressed in all cell lines, including SCLC, ADC, and SCC, and the expression pattern of Draxin resembles that of Netrin-1. Contrary to the histological type-independent expression of ligands, the expression of individual receptors is varied-DCC is expressed more in non-SCLC cell lines, and Neogenin is expressed more in SCLC cell lines. The difference in receptor expression patterns in different histological cell types may be important to understand the biological properties of lung cancer cells from the view point of cancer cell regulation through guidance molecule-receptor interactions. Expression of Draxin, Netrin-1, DCC and Neogenin is not always similar between the lung cancer cell lines with WB analysis and the formalin-fixed and paraffin-embedded lung cancer tissues with IHC analysis. We suspect that the difference in results could be attributed to the difference in sample preparing methods and in environments of cancer cell; culture medium in the cancer cell lines and extracellular matrix in the cancer tissues.

Draxin can be a link to receptors, such as DCC, Neogenin, UNK5 and DSCAM [1], and can connect to Netrin-1 through 22aa. This means that Draxin can be related to tumorigenesis, metastasis, and invasion similarly to Netrin-1. Netrin-1 is associated with tumor progression by inducing apoptosis in metastatic breast cancer and metastatic colorectal cancer $[12,24]$. Netrin-1/DCC interaction mediates tumor progression by inducing apoptosis in diffuse large B-cell lymphoma (DLBCL) and mantle cell lymphoma (MCL). In addition, Netrin-1 antibody inhibits the interaction of Netrin-1 and DCC in vitro and vivo, and Netrin-1 acts as a survival factor for activated B cells in DLBCL and MCL tumor cells [5]. Furthermore, Netrin-1/ Neogenin interaction promotes medulloblastoma cell invasion, angiogenesis, and gastric cancer cell proliferation and 


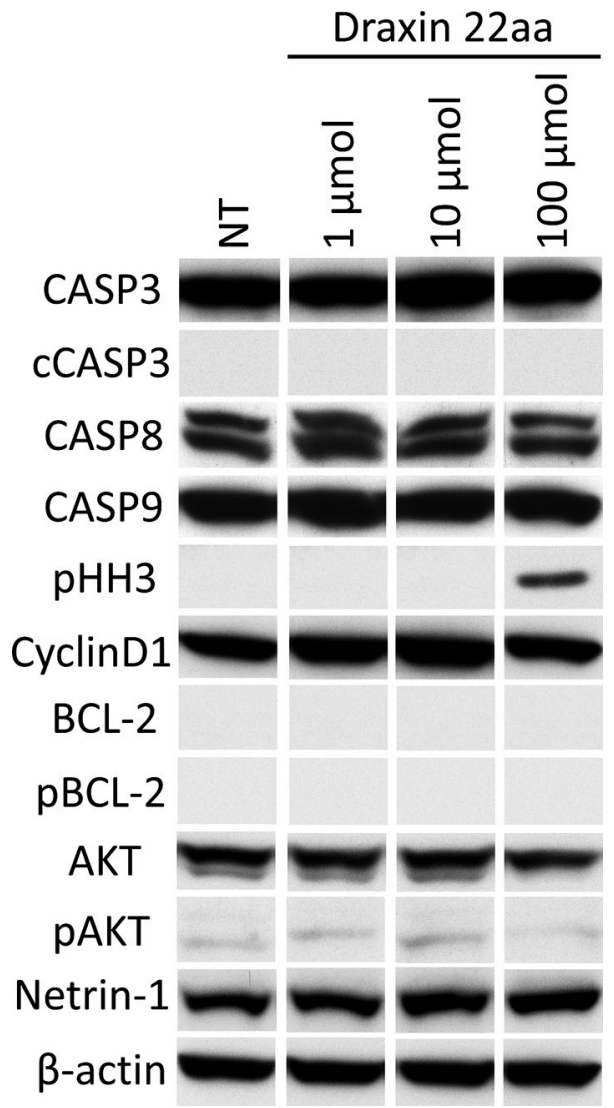

Fig. 5. Western blot analysis of adenocarcinoma cells (H358) treated with Draxin 22-amino-acid peptide. Four biological replicates were analyzed. Representatives of no treatment (NT), and treatment treated with Draxin 22-amino acid peptide in an amount of $1 \mu \mathrm{mol}, 10 \mu \mathrm{mol}$ and $100 \mu \mathrm{mol}$ are shown. Reactivity of antibody markers are consistent with western blot analysis of Draxin knockdown in H358. invasion $[2,35]$. To demonstrate the biological significance of Draxin, knockdown of Draxin mRNA by siRNA was performed in H358 adenocarcinoma. Delloye-Bourgeois did Netrin-1 SiRNA experiment using the same adenocarcinoma cell line, and reported that suppression of Netrin-1 enhanced tumor cell apoptosis in the cell [8]. On the contrary, suppression of Draxin expression decreased apoptosis-regulating cysteine proteases, such as Caspase3, Caspase8, and Caspase9, and increased phosphorylated histone $\mathrm{H} 3$ (ser10), a mitosis marker. In addition, we demonstrated that the Ki-67 labeling index is upregulated in H358 cells after reduction of Draxin. These data suggest that Draxin can modulate cell survival and proliferation of lung cancer cells.

Another characteristic of Draxin is the ability to bind directly to Netrin-1 in the absence of guidance molecule receptors. The 22aa of Draxin binds to the third EGF domain of Netrin-1, and Draxin can modulate Netrin-1/ receptor signaling [15]. To clarify the effects of Draxin 22aa on lung cancer cells, we synthesized Draxin 22aa and added the peptide to an adenocarcinoma cell line H358. Although we hypothesized that the peptide might induce apoptosis by inhibiting Netrin-1, expression of Caspase3, Caspase8, and Caspase9 was not altered in H358 cells treated with peptide. However, in lung cancer cells, 22aa peptide increased expression of phosphorylated histone H3, similar to that observed in Draxin-knockdown H358 cells. This suggests that guidance molecules and their receptors are present in lung cancer cells because Draxin shares guidance molecule receptors with Netrin-1 [1] and interacts directly with Netrin-1 [15]. However, the detailed mechanism of cancer cell modification by Draxin needs further study.

Table 3. NNK-induced lung tumors in Draxin-deficient mice

\begin{tabular}{lccccc}
\hline Gene status & No. of mice & No. of tumor-bearing mice & Total tumor number & Total adenoma number & Total adenocarcinoma number \\
\hline Wild-type & 10 & 6 & 8 & 5 & 3 \\
Draxin null & 10 & 6 & 7 & 5 & 2
\end{tabular}

\section{Wild mouse}

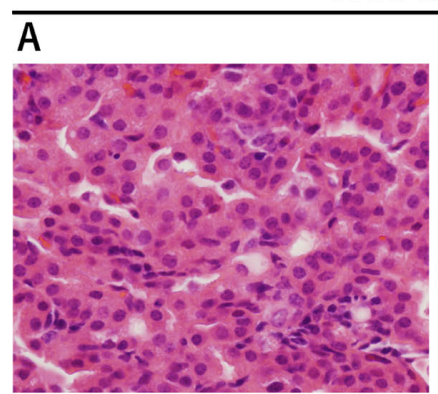

Draxin deficient mouse
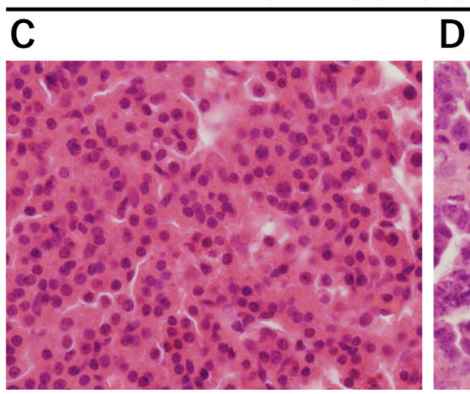

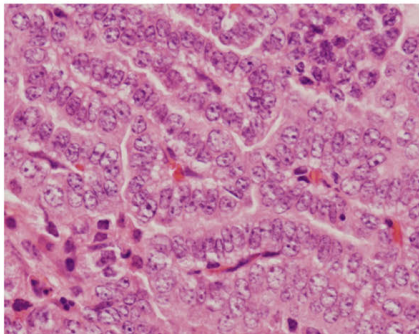

D

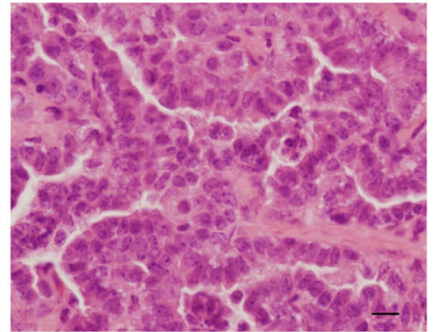

Fig. 6. NNK-induced lung tumors in wild and Draxin gene-deficient mice. (A) Adenoma in a wild mouse. (B) Adenocarcinoma in a wild mouse is well to moderately-differentiated papillary type. Tumor size was about $2 \mathrm{~mm}$. (C) Adenoma in a Draxin gene-deficient mouse. (D) Adenocarcinoma in a Draxin gene-deficient mouse is well to moderately-differentiated papillary type. Tumor size was about $2 \mathrm{~mm}$. Bar $=20 \mu \mathrm{m}$. 
To understand the relationship of Draxin and tumorigenesis in vivo, we treated wild-type and Draxin genedeficient mice with NNK. However, the tumor size and the total number of lung tumors, including the number of lung adenoma and adenocarcinoma, was not significantly different between wild-type and Draxin gene-deficient mice. In case of adenocarcinoma, differentiation grade was well to moderately in both mice. Draxin deficiency alone was not sufficient to modify NNK-induced tumorigenesis. In Draxin gene-deficient mice, no lung morphological abnormalities were manifested (data not shown), which suggests that Draxin-mediated tumorigenesis requires additional factors.

\section{Conclusions}

Draxin is expressed in lung cancer cells, and Draxin knockdown in an adenocarcinoma cell line suggests that Draxin might be involved in cell proliferation and apoptosis of lung adenocarcinoma cells. However, our mouse tumorigenesis study suggests that Draxin requires additional factors for tumorigenesis in vivo. Further studies are needed to investigate the significance of Draxin in lung cancer and the role of Draxin-receptor and Draxin-Netrin-1 interactions in tumorigenesis.

\section{Conflicts of Interest} interest.

The authors declare that they have no conflict of

\section{Acknowledgments}

This study was partially supported by a Grant-in-Aid for Scientific Research (16K15459) from the Ministry of Education, Culture, Sports, Science and Technology of Japan. And, this study was also supported in part by a grant from the Smoking Research Foundation.

We thank Mrs. Motoko Kagayama and Mrs. Takako Maeda for their skillful technical assistance.

\section{References}

1. Ahmed, G., Shinmyo, Y., Ohta, K., Islam, S., Hossain, M., Naser, I., Riyadh, M., Su, Y., Zhang, S., Tessier-Lavigne, M. and Tanaka, H. (2011) Draxin Inhibits Axonal outgrowth through the netrin receptor DCC. J. Neurosci. 31; 14018-14023.

2. Akino, T., Han, X., Nakayama, H., McNeish, B., Zurakowski, D., Mammoto, A., Klagsbrun, M. and Smith, E. (2014) Netrin-1 promotes medulloblastoma cell invasiveness and angiogenesis, and demonstrates elevated expression in tumor tissue and urine of patients with pediatric medulloblastoma. Cancer Res. 74; 3716-3726.

3. Arakawa, H. (2004) Netrin-1 and its receptors in tumorigenesis. Nat. Rev. Cancer 4; 978-987.

4. Bredseen, D., Mehlen, P. and Rabizadeh, S. (2004) Apoptosis and dependence receptors: a molecular basis for cellular addiction. Physiol. Rev. 84; 411-430.

5. Broutier, L., Creveaux, M., Vial, J., Tortereau, A., Delcros, J., Chazot, G., Mccarron, M., Léon, S., Pangault, C., Gadot, N., Colombe, A., Boulland, M., Blachier, J., Marie, J., Traverseglehen, A., Donzé, O., Chassagne-clément, C., Salles, G., Tarte, K., Mehlen, P. and Castets, M. (2016) Targeting netrin-1 / DCC interaction in diffuse large B-cell and mantle cell lymphomas. EMBO Mol. Med. 8; 96-104.

6. Castets, M., Coissieux, M., Delloye-Bourgeois, C., Bernard, L., Delcros, J., Bernet, A., Laudet, V. and Mehlen, P. (2009) Inhibition of endothelial cell apoptosis by netrin-1 during angiogenesis. Dev. Cell 16; 614-620.

7. Dalvin, S., Anselmo, M., Prodhan, P., Komatsuzaki, K., Schnitzer, J. and Kinane, T. (2003) Expression of Netrin-1 and its two receptors DCC and UNC5H2 in the developing mouse lung. Gene Expr. Patterns 3; 279-283.

8. Delloye-Bourgeois, C., Brambilla, E., Coissieux, M., Guenebeaud, C., Pedeux, R., Firlej, V., Cabon, F., Brambilla, C., Mehlen, P. and Bernet, A. (2009) Interference with netrin-1 and tumor cell death in non-small cell lung cancer. J. Natl. Cancer Inst. 101; 237-247.

9. Dickson, B. (2002) Molecular mechanisms of axon guidance. Dev. Biol. 298; 1959-1964.

10. Dumartin, L., Quemener, C., Laklai, H., Herbert, J., Bicknell, R., Bousquet, C., Pyronnet, S., Castronovo, V., Schiling, M., Bikfalvi, A. and Hagedorn, M. (2010) Netrin-1 mediates early events in pancreatic adenocarcinoma progression, acting on tumor and endothelial cells. Gastroenterology 138; 1595-1606.

11. Fearon, E., Cho, K., Nigro, J., Kern, S., Simons, J., Ruppert, J., Preisinger, A., Thomas, G., Kinzler, K. and Et, A. (1990) Identification of a chromosome $18 \mathrm{q}$ gene that is altered in colorectal cancers. Science 247; 49-56.

12. Fitamant, J., Guenebeaud, C., Coissieux, M., Guix, C., Treilleux, I., Scoazec, J., Bachelot, T., Bernet, A. and Mehlen, P. (2008) Netrin-1 expression confers a selective advantage for tumor cell survival in metastatic breast cancer. Proc. Natl. Acad. Sci. US A $105 ; 4850-4855$.

13. Fitzgerald, D., Seaman, C. and Cooper, H. (2006) Localization of neogenin protein during morphogenesis in the mouse embryo. Dev. Dyn. 235; 1720-1725.

14. Foley, J., Anderson, M., Stoner, G., Gaul, B., Hardsity, J. and Marnopot, R. (1991) Proliferative lesions of the mouse lung: progression studies in strain A mice. Exp. Lung Res. 17; $157-$ 168.

15. Gao, X., Metzger, U., Panza, P., Mahalwar, P., Alsheimer, S., Geiger, H., Maischein, H., Levesque, M., Templin, M. and Söllner, S. (2015) A floor-plate extracellular protein-protein interaction screen identifies Draxin as a secreted Netrin-1 antagonist. Cell Rep. 12; 694-708.

16. Goshima, Y., Ito, T., Sasaki, Y. and Nakamura, F. (2002) Semaphorins as signals for cell repulsion and invasion. J. Clin. Invest. 109; 993-998.

17. Hinck, L. (2004) The versatile roles "axon guidance" cues in tissue morphogenesis. Dev. Cell 7; 783-793.

18. Islam, S., Shinmyo, Y., Okafuji, T., Su, Y., Neser, I., Ahmed, G., Zhang, S., Chen, S., Ohta, K., Kiyonari, H., Abe, T., Tanaka, S., Nishinakamura, R., Terashima, T., Kitamura, T. and Tanaka, H. (2009) Draxin, a repulsive guidance protein for spinal cord and forebrain commissures. Science 323; 388-393.

19. Ito, T., Kagoshima, M., Sasaki, Y., Li, C., Udaka, N., Kitsukawa, T., Fujisawa, H., Taniguchi, M., Yagi, T., Kitamura, H. and Goshima, Y. (2000) Repulsive axon guidance molecule Sema3A inhibits branching morphogenesis of fetal mouse lung. Mech. Dev. 97; 35-45

20. Kagoshima, M., Ito, T., Kitamura, H. and Goshima, Y. (2001) 
Diverse gene expression and function of semaphorins in developing lung: Positive and negative regulatory roles of semaphorins in lung branching morphogenesis. Genes Cells 6; 559-571.

21. Kanda, Y. (2013) Investigation of the freely-available easy-to-use software "EZR" (Easy R) for medical statistics. Bone Marrow Transplant. 48; 452-458.

22. Kaufmann, S., Kuphal, S., Schubert, T. and Bosserhoff, A. (2009) Functional implication of Netrin expression in malignant melanoma. Cell. Oncol. 31; 415-422.

23. Keino-Masu, K., Masu, M., Hinck, L., Leonardo, E., Chan, S., Culotti, J. and Tessier-Lavigne, M. (1996) Deleted in Colorectal Cancer (DCC) encodes a netrin receptor. Cell 87; 175-185.

24. Ko, S., Blatch, G. and Dass, C. (2014) Netrin-1 as a potential target for metastatic cancer: Focus on colorectal cancer. Cancer Metastasis Rev. 33; 101-113.

25. Liu, Y., Stein, E., Oliver, T., Li, Y., Brunken, W., Koch, M., Tessier-Lavigne, M. and Hogan, B. (2004) Novel role for netrins in regulating epithelial behavior during lung branching morphogenesis. Curr. Biol. 14; 897-905.

26. Llambi, F., Lourenco, F., Gozuacik, D., Guix, C., Pays, L., Del Rio, G., Kimchi, A. and Mehlen, P. (2005) The dependence receptor UNC5H2 mediates apoptosis through DAP-kinase. EMBO J. 24; 1192-1201.

27. Lu, X., le Noble, F., Yuan, L., Jiang, Q., de Lafarge, B., Sugiyama, D., Breant, C., Claes, F., De Smet, F., Thomas, J., Autiero, M., Carmeliet, P., Tessier-Lavigne, M. and Eichmann, A. (2004) The netrin receptor UNC5B mediates guidance events controlling morphogenesis of the vascular system. Nature 432; 179-186.

28. Mehlen, P., Delloye-Bourgeosis, C. and Chedotal, A. (2011)
Novel roles for Slits and netrins: axon guidance cues as anticancer targets? Nat. Rev. Cancer 11; 188-197.

29. Mille, F., Thibert, C., Fombonne, J., Rama, N., Guix, C., Hayashi, H., Corset, V., Reed, J. and Mehlen, P. (2009) The Patched dependence receptor triggers apoptosis through a DRAL-caspase-9 complex. Nat. Cell Biol. 11; 739-746.

30. Nasarre, P., Potiron, V., Drabkin, H. and Roche, J. (2010) Guidance molecules in lung cancer. Cell Adh. Migr. 4; 130-145.

31. Serafini, T., Colamarino, S., Leonardo, E., Wang, H., Beddington, R., Skarnes, W. and Tessier-Lavigne, M. (1996) Netrin-1 is required for commissural axon guidance in the developing vertebrate nervous system. Cell 87; 1001-1014.

32. Suchting, S., Bicknell, R. and Eichman, A. (2006) Neuronal clues to vascular guidance. Exp. Cell Res. 312; 668-675.

33. Tessier-Lavigne, M. and Goodman, C. (1996) The molecular biology of axon guidance. Science 274; 1123-1133.

34. Xu, K., Wu, Z., Renier, N., Antipenko, A., Tzvetkova-Robev, D., Xu, Y., Minchenko, M., Nardi-Dei, V., Rajashankar, K., Himanen, J., Tessier-Lavigne, M. and Nikolov, D. (2014) Structures of netrin- 1 bound to two receptors provide insight into its axon guidance mechanism. Science 344; 1275-1279.

35. Yin, K., Wang, L., Zhang, X., He, Z., Xia, Y., Xu, J., Wei, S., Li, B., Li, Z., Sun, G., Li, Q., Xu, H. and Xu, Z. (2017) Netrin-1 promotes gastric cancer cell proliferation and invasion via the receptor neogenin through PI3K/AKT signaling pathway. Oncotarget 8; 51177-51189.

36. Zou, Y. and Lyuksyutova, A. (2007) Morphogens as conserved axon guidance cues. Curr. Opin. Neurobiol. 17; 22-28.

This is an open access article distributed under the Creative Commons Attribution License, which permits unrestricted use, distribution, and reproduction in any medium, provided the original work is properly cited. 